\title{
Accelerating the discovery of multilayer nanostructures with analytic differentiation of the transfer matrix equations
}

\author{
James F. Varner, Dayanara Wert, Aya Matari, Raghad Nofal, and Jonathan J. Foley IV ๑* \\ Department of Chemistry, William Paterson University, 300 Pompton Road, Wayne, New Jersey 07470, USA
}

(Received 10 September 2019; published 7 January 2020)

\begin{abstract}
Multilayer nanostructures represent an important class of materials with tunable optical and thermal radiative properties that can be leveraged for a wide range of energy applications. We present a theoretical framework for optimizing the geometry of such structures that utilizes gradients of various objective functions that are enabled through analytic differentiation of the transfer-matrix equations. We demonstrate the usefulness of this method by applying it to the local optimization of many-degree-of-freedom structures for incandescent light sources, and the global optimization of few-degree-of-freedom structures that serve as solar cell coatings and optical cavities for enhancing the absorption of organic chromophores embedded in thin films.
\end{abstract}

DOI: 10.1103/PhysRevResearch.2.013018

\section{INTRODUCTION}

Designing materials on the nanoscale can have a profound impact on how optical energy flows through those materials, which can in turn dramatically improve the performance of nanostructured materials for energy-related applications including solar and (solar) thermophotovoltaic energy conversion [1-17], radiative cooling [18-23], incandescent lighting [24-27], among others. Multilayer planar nanomaterials, stacks of flat materials with nanoscale thickness, have emerged as promising candidates for such applications because they present highly tunable optical and thermal emission properties and are relatively easy to fabricate. As seemingly simple as such structures are, the spectral shaping capabilities they offer have enabled passive cooling well below ambient temperature [19-23], and incandescent lighting with efficiencies meeting or exceeding LED bulbs [27]. Also important is that the optical and thermal emission properties of such structures can be efficiently simulated; for example, several of the authors have developed an open-source package called WPTherml [28] that is based on an efficient analytic method for solving Maxwell's equations for such structures known as the transfer-matrix method (TMM) [29].

The efficient simulation of optical properties of multilayer planar nanostructures, through TMM or related methods, has been leveraged for a variety of different design approaches for multilayer nanostructures including genetic algorithms [30], multilevel single-linkage optimizations [4,31], and ab initio inverse design [32] methods. Gradients, i.e., partial derivatives of an objective function with respect to system variables,

\footnotetext{
*foleyj10@wpunj.edu

Published by the American Physical Society under the terms of the Creative Commons Attribution 4.0 International license. Further distribution of this work must maintain attribution to the author(s) and the published article's title, journal citation, and DOI.
}

provide critical information about how to systematically search system variables for local optima, and can greatly enhance the efficiency of global optimization techniques that rely on multiple local optimizations $[4,33,34]$. Here we provide a simple and efficient route to the generation of such gradients through analytic differentiation of the transfer-matrix equations that provides an efficient engine for generating exact gradients for objective functions that derive from optical and/or thermal radiation properties. This theoretical advance therefore provides a powerful tool for accelerating the computational design and discovery of multilayer nanostructures for a variety of applications.

Here we focus specifically on applications of multilayer nanostructures whose performance derives from the optical properties of reflectivity, transmissivity, absorptivity/ emissivity, and by extension, thermal radiation. In such cases, one can vary the number of discrete layers that compose the multilayer, the materials that compose each discrete layer, and the thickness of each discrete layer to affect the desired change in optical and thermal radiation properties. Even within practical fabrication constraints, the search space of all such multilayers is unmanageably vast. In this work, we address only the variability of the thickness of each discrete layer (where we denote the thickness of layer $i$ by $s_{i}$ ) in a multilayer whose total number and composition of discrete layers is fixed. We derive equations for the analytical derivatives of the transfer-matrix equations with respect to layer thicknesses $\left(s_{i}\right)$ and develop a framework for connecting these derivatives to objective functions to be optimized over. We present a detailed analysis of the efficiency of this methodology as compared to one based on numerical gradients. The resulting methodology is applied to the local optimization of 90-layer filters for incandescent light sources, identifying three different structures with predicted luminous efficiency exceeding 30\%. Finally, this analytic gradient engine is coupled to a basin-hopping algorithm and used to identify globally optimum coatings for thin-film photovoltaic and cavities for enhanced light harvesting. We believe this methodology provides a powerful 
enabling tool for the design and analysis of multilayer nanomaterials in a number of domains including photonics, materials science, plasmonics, nanoscience, energy research, and chemical physics.

\section{THEORY}

As illustrative objective functions, consider two generic figures of merit for thermal radiation applications: the first $(\rho)$ will be a dimensional figure of merit and will take the form of a density (for example, a power density for radiative cooling applications [18-23]), and the second $(\eta)$ will be dimensionless figure of merit and will take the form of an efficiency (for example, the luminous efficiency for incandescent light sources [24-27]). The main ideas apply also to figures of merit which depend only upon optical properties; we introduce and optimize several such figures of merit in the Results section. We can express $\rho$ as an integral over the product of the thermal emission and some spectral function related to the application, which we will denote as $v(\lambda)$ throughout. Because the thermal emission spectrum is itself a product of the emissivity spectrum and Planck's blackbody law $[B(\lambda, T)]$, we can write such a figure of merit as

$$
\rho=\int_{\lambda_{\min }}^{\lambda_{\max }} v(\lambda) B(\lambda, T) \epsilon(\lambda) d \lambda,
$$

where only the emissivity spectrum $\epsilon(\lambda)$ depends upon materials/geometry of the emitting structure. We can express $\eta$ as a density normalized by the total thermal emission:

$$
\eta=\frac{\int_{\lambda_{\min }}^{\lambda_{\max }} v(\lambda) B(\lambda, T) \epsilon(\lambda) d \lambda}{\int_{\lambda_{\min }}^{\lambda_{\max }} B(\lambda, T) \epsilon(\lambda) d \lambda} .
$$

We will denote the geometric degrees of freedom (i.e., the layer thicknesses) by the vector $\mathbf{s}$, so the elements of the gradient vector can be written as

$$
\frac{\partial \rho}{\partial s_{i}}=\int_{\lambda_{\min }}^{\lambda_{\max }} v(\lambda) B(\lambda, T) \frac{\partial \epsilon(\lambda)}{\partial s_{i}} d \lambda,
$$

and

$$
\frac{\partial \eta}{\partial s_{i}}=\frac{\frac{\partial \rho}{\partial s_{i}} P-\frac{\partial P}{\partial s_{i}} \rho}{P^{2}}
$$

where $P$ indicates the denominator of Eq. (2) and

$$
\frac{\partial P}{\partial s_{i}}=\int_{\lambda_{\min }}^{\lambda_{\max }} B(\lambda, T) \frac{\partial \epsilon(\lambda)}{\partial s_{i}} d \lambda .
$$

Conveniently, only the emissivity depends upon $\mathbf{s}$, and so the key to be able to differentiate $\epsilon(\lambda)$ analytically with respect to each geometric parameter, $s_{i}$.

The emissivity (which we take to be equivalent to the absorptivity by Kirchoff's law) at a particular wavelength can be written in terms of the reflectivity and transmissivity,

$$
\epsilon=1-R-T,
$$

where $R$ and $T$ are the reflectivity and transmissivity, respectively. Both $R$ and $T$ can be written as products of complex amplitudes that are determined directly from the transfer matrix, namely

$$
R=r r^{*}
$$

and

$$
T=t t^{*} \frac{n_{L} \cos \left(\theta_{L}\right)}{n_{1} \cos \left(\theta_{1}\right)},
$$

where $\theta_{1}\left(\theta_{L}\right)$ indicate the angle of incidence (refraction) into the top-most (terminal) layers of the multilayer. Differentiating the emissivity therefore leads to the following expression:

$$
\frac{\partial \epsilon}{\partial s_{i}}=-\frac{\partial R}{\partial s_{i}}-\frac{\partial T}{\partial s_{i}},
$$

where the partial derivatives of the reflectivity and transmissivity have the forms

$$
\frac{\partial R}{\partial s_{i}}=\frac{\partial r}{\partial s_{i}} r^{*}-r \frac{\partial r^{*}}{\partial s_{i}}
$$

and

$$
\frac{\partial T}{\partial s_{i}}=\left(\frac{\partial t}{\partial s_{i}} t^{*}-t \frac{\partial t^{*}}{\partial s_{i}}\right) \frac{n_{L} \cos \left(\theta_{L}\right)}{n_{1} \cos \left(\theta_{1}\right)},
$$

respectively. The reflectivity and transmissivity amplitudes ( $r$ and $t$, respectively) derive from the elements of the transfer matrix itself:

$$
\begin{aligned}
& r=\frac{M_{21}}{M_{11}}, \\
& t=\frac{1}{M_{11}},
\end{aligned}
$$

and $r^{*}$ and $t^{*}$ are the complex conjugates of $r$ and $t$, respectively. Again, applying the chain rule, we get

$$
\frac{\partial}{\partial s_{i}} r=\frac{M_{11} \frac{\partial M_{21}}{\partial s_{i}}-M_{21} \frac{\partial M_{11}}{\partial s_{i}}}{M_{11}^{2}}
$$

and

$$
\frac{\partial}{\partial s_{i}} t=-\frac{\frac{\partial M_{11}}{\partial s_{i}}}{M_{11}^{2}},
$$

where the elements of the transfer matrix are defined as

$$
\left(\begin{array}{ll}
M_{11} & M_{12} \\
M_{21} & M_{22}
\end{array}\right)=\mathbf{D}_{1}^{-1}\left(\prod_{l=2}^{L-1} \mathbf{D}_{l} \mathbf{P}_{l} \mathbf{D}_{l}^{-1}\right) \mathbf{D}_{L} .
$$

Explicit expressions for the $\mathbf{D}$ and $\mathbf{P}$ matrices can be found in Appendix A, and importantly, only matrix $\mathbf{P}_{i}$ depends on the thickness of layer $s_{i}$. Hence, the derivatives of the transfermatrix elements with respect to layer thicknesses are easy to compute:

$$
\begin{aligned}
& \left(\begin{array}{ll}
\frac{\partial M_{11}}{\partial s_{i}} & \frac{\partial M_{12}}{\partial s_{i}} \\
\frac{\partial M_{21}}{\partial s_{i}} & \frac{\partial M_{22}}{\partial s_{i}}
\end{array}\right) \\
& =\mathbf{D}_{1}^{-1}\left(\prod_{l=2}^{i-1} \mathbf{D}_{l} \mathbf{P}_{l} \mathbf{D}_{l}^{-1}\right) \mathbf{D}_{i} \frac{\partial \mathbf{P}_{i}}{\partial s_{i}} \mathbf{D}_{i}^{-1}\left(\prod_{l=i+1}^{L-1} \mathbf{D}_{l} \mathbf{P}_{l} \mathbf{D}_{l}^{-1}\right) \mathbf{D}_{L},
\end{aligned}
$$

where

$$
\frac{\partial \mathbf{P}_{i}}{\partial s_{i}}=\left(\begin{array}{cc}
-i k_{z, i} \exp \left(-i k_{z, i} s_{i}\right) & 0 \\
0 & i k_{z, i} \exp \left(i k_{z, i} s_{i}\right)
\end{array}\right) .
$$


Thus, the general prescription for computing the gradient of an objective function of the form Eq. (3) or Eq. (4) is to

(i) compute the gradient of the transfer matrix via Eq. (17);

(ii) compute the gradients of the reflectivity and transmissivity amplitudes via Eqs. (14) and (15), and the gradients of the reflectivity and transmissivity via Eqs. (10) and (11);

(iii) compute the gradients of the emissivity via Eq. (9);

(iv) utilize the gradient of the emissivity to compute Eq. (3) or Eq. (4).

It is important to note that this procedure can be seamlessly adapted to figures of merit that depend on the reflectivity and/or transmissivity rather than the emissivity; one simply utilizes the reflectivity gradient and/or the transmissivity gradient.

\section{RESULTS AND DISCUSSION}

We have implemented the analytic gradient formalism in a development version of WPTherml [28], which has permitted careful testing of its efficiency as compared to a numerical gradient formalism based on centered finite differences. We have also applied our analytic gradient formalism to the geometry optimization of multilayer structures for (a) selective thermal emission and filtration for incandescent lighting, (b) enhanced solar absorption of silicon-based PV films, and (c) enhanced optical absorption of organic dyes embedded in thin films. For application (a), we demonstrate local optimizations of multilayer filters with 90 degrees of freedom, leading to a nearly twofold improvement in luminous efficiency compared to unoptimized filters and nearly a tenfold improvement compared to unfiltered emitters. For applications (b) and (c), we perform global optimizations over a diverse set of multilayers, albeit with more modest degrees of freedom compared to application (a). In each case, we report timings compared to analogous optimizations utilizing numerical gradients to highlight the advantage of our analytical gradient formulation (see Fig. 1).

In all three both applications, the bounded Limitedmemory Broyden-Fletcher-Goldfarb-Shanno [33] method (i.e., the L-BFGS-B method) is used to perform local geometry optimizations; application (a) is treated as a local optimization problem only, and in (b) and (c) multiple local L-BFGS-B optimizations are utilized in a global optimization technique known as the basin hopping method $[35,36]$. In both applications, the L-BFGS-B geometry updates constitute the rate limiting step of the optimization. These geometry updates are performed according to

$$
\Delta \mathbf{s}=\mathbf{B g},
$$

where $\Delta \mathbf{S}$ is a geometry update vector, $\mathbf{g}$ is the gradient vector, and $\mathbf{B}$ is an approximation to the inverse of the Hessian matrix that is iteratively formed in L-BFGS method [33]. The elements of $\mathbf{g}$ take the form of integrals involving derivatives of optical quantities, such as the emissivity as shown in Eqs. (3) and (4), and so our analytic gradient methodology directly accelerates local geometry updates through the acceleration of computing the gradient vector. It should also be mentioned that numerical gradients are approximations to analytic gradients and therefore may lack accuracy in some cases; however, empirically we have found the two agree

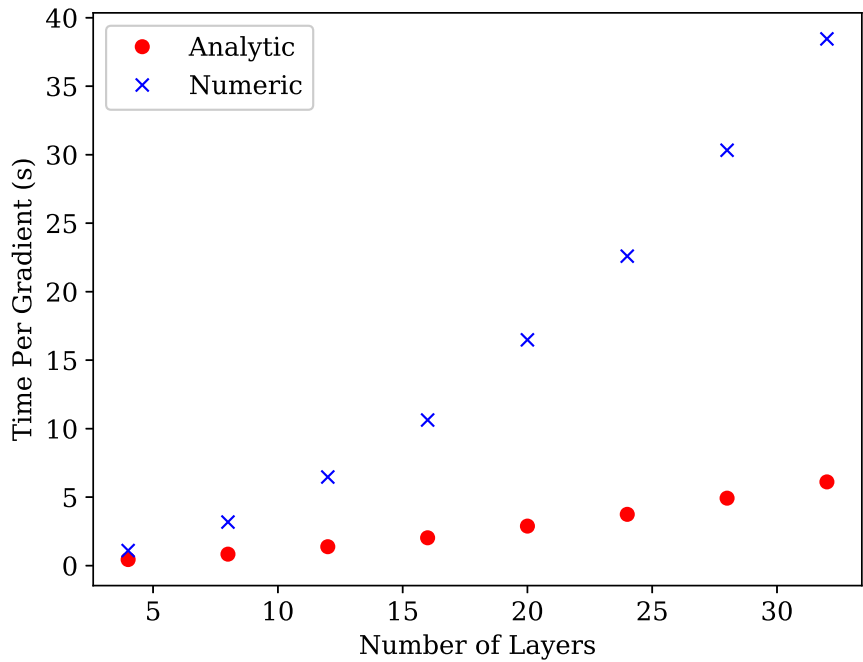

FIG. 1. Comparison of average time to compute the gradient vector for multilayer structures as a function of the number of layers using analytic expressions compared to numeric differentiation using centered finite differences. Timings were performed on a iMac running macOS High Sierra with a 3.6-GHz Intel Core i7.

to high precision in a large number of cases. Notably, we have confirmed that all optimizations presented in this paper converge to the same solution using analytic and numeric gradients, and that in all cases analytic solutions provide more expeditious convergence.

To understand this advantage, it is important to realize that the main computational bottleneck in computing the figures of merit given in Eqs. (1) and (2) is the computation of the optical properties [e.g., $\epsilon(\lambda)]$ across the wavelength range spanning $\lambda_{\min }$ to $\lambda_{\max }$, typically with a numerical resolution $d \lambda \approx 1 \mathrm{~nm}$, meaning typically thousands of $\epsilon(\lambda)$ values must be computed to enable computation of Eqs. (1) and (2). Each value of $\epsilon(\lambda)$ represents a unique solution to the transfermatrix equations where, as can be seen in Eq. (16), the main computational effort lies in forming and multiplying the $3 L$ matrices $\left(\mathbf{D}_{l}, \mathbf{P}_{l}, \mathbf{D}_{l}^{-1}\right)$ where $l$ denotes an individual layer of the structure and $L$ is the total number of layers. Similarly, in the computation of the gradients of the figures of merit in Eqs. (3) and (4), the terms involving $\frac{\partial \epsilon(\lambda)}{\partial s_{i}}$ represent unique derivatives of the transfer matrix for each wavelength. A key theoretical insight motivating this work is that only the $\mathbf{P}$ matrices have dependence on the geometry of the multilayer structures, and therefore the efficiency advantage of analytic gradients derives from the fact that they preserve linear scaling with $L$ in the number of matrices that must be built to compute the gradients, whereas numerical gradients lead to quadratic scaling with $L$ in the number of matrices that must be built.

Consider the simplest approximation for the numeric gradient of Eq. (1); each element of the gradient is given by

$$
\frac{\partial \rho}{\partial s_{i}}=\frac{\rho\left(\mathbf{s}+\delta_{i}\right)-\rho\left(\mathbf{s}-\delta_{i}\right)}{2 \delta},
$$

where $\mathbf{s}$ is the vector of thicknesses of each of the $L$ layers in the multilayer and $\delta_{\mathbf{i}}$ is an $L$-dimensional vector that is filled with zeros except for entry $i$, which has the value $\delta$. To compute the gradient in this fashion, the transfer-matrix 
TABLE I. Timings for computing matrices required by transfer matrix-method compared to cost of multiplying matrices. Averages are taken over 100 different computations for multilayers with $L$ layers. Timed on a iMac running macOS High Sierra with a $3.6 \mathrm{GHz}$ Intel Core i7.

\begin{tabular}{lccccc}
\hline \hline$L$ & $\begin{array}{c}\text { Build D } \\
(\mu \mathrm{s})\end{array}$ & $\begin{array}{c}\text { Build } \mathbf{P} \\
(\mu \mathrm{s})\end{array}$ & \begin{tabular}{c}
${\text { Build } \mathbf{D}^{-1}}_{(\mu \mathrm{s})}$ \\
\hline 4
\end{tabular} & $\begin{array}{c}\text { Total build } \\
(\mu \mathrm{s})\end{array}$ & $\begin{array}{c}\text { Multiply } \\
(\mu \mathrm{s})\end{array}$ \\
\hline 8 & 68.70 & 28.06 & 9.903 & 75.68 & 15.58 \\
16 & 127.0 & 49.16 & 18.42 & 136.2 & 29.04 \\
32 & 248.2 & 168.3 & 32.22 & 245.9 & 51.00 \\
\hline \hline
\end{tabular}

equations at a given wavelength must be solved twice for each of the $L$ degrees of freedom. Consequently, the number of matrices that must be formed grows proportional to $L^{2}$ and the number of matrix multiplications also grows proportional to $L^{2}$. To compute the derivative analytically, the number of matrix multiplications required still grows proportional to $L^{2}$, but only one additional matrix $\left(\frac{\partial \mathbf{P}}{\partial s_{i}}\right)$ must be formed per degree of freedom, so the number of matrices that must be formed grows only as $L$. As we shown in Table I, building the $\mathbf{D}, \mathbf{P}$, and $\mathbf{D}^{-1}$ matrices actually constitutes the main computational bottleneck of the transfer-matrix method, so retaining linear scaling in this step represents a significant advantage for analytical gradients over numerical gradients. In particular, for several representative multilayers, we find that average time to build all of the $\mathbf{D}, \mathbf{P}$, and $\mathbf{D}^{-1}$ matrices is roughly five times longer than the time required to perform all matrix multiplications between them (see Table I). To further highlight the advantage of our analytical formulation, we repeat 100 different computations of the total gradient vector for several representative multilayers (having between $L=4$ and $L=32$ layers) according to Eq. (3) with $\lambda_{\min }=300 \mathrm{~nm}$, $\lambda_{\max }=4000 \mathrm{~nm}, d \lambda=3.7 \mathrm{~nm}$ using both analytical numeric derivatives. We find that the advantage of using analytic gradients grows substantially with the number of layers, with the numeric gradients being approximately 2.5 times slower when $L=4$ and six times slower when $L=32$ (see Fig. 1).

\section{A. Optimization of incandescent emitters and filters}

Incandescent light sources utilize the thermal radiation of a hot (2500-3000 K) emitter, usually made from thermally stable metals like tungsten [24-27]. Although incandescent sources provide bright and pleasing light, their energy efficiency is low owing to their broad emission that falls largely outside the visible part of the spectrum. A key figure of merit for incandescent sources is the luminous efficiency, which quantifies the fraction of thermal emission that falls within the human eye's sensitivity window (quantified by the so-called photopic luminosity function) to the total emitter power,

$$
\eta_{\text {lum }}=\frac{\int_{0}^{\infty} B(\lambda, T) \epsilon(\lambda) v(\lambda) d \lambda}{\int_{0}^{\infty} B(\lambda, T) \epsilon(\lambda) d \lambda},
$$

where $v(\lambda)$ is the photopic luminosity function and $\epsilon(\lambda)$ is the emissivity spectrum of the emitter. Several researchers have demonstrated the promise of engineered multilayers as incandescent sources that can reflect IR thermal radiation back to the emitter, and transmit visible light to the observer [25,27]. Such a design involves an emitter (which may or may not be an engineered structure) and a filter which is specifically engineered to be a selective transmitter and reflector, as shown in Fig. 2(a). A simple modification can be made to the figure of merit in Eq. (21) to account for the impact of the filter as follows:

$$
\eta_{\text {lum }}^{e+F}=\frac{\int_{0}^{\infty} B\left(\lambda, T_{e}\right) \epsilon_{e}(\lambda) T_{F}(\lambda) v(\lambda) d \lambda}{\int_{0}^{\infty} B\left(\lambda, T_{e}\right) \epsilon_{e}(\lambda) T_{F}(\lambda) d \lambda},
$$

where $\epsilon_{e}(\lambda)$ is the emissivity of the emitter, $T_{F}(\lambda)$ is the transmissivity of the filter, and $T_{e}$ specifically denotes the temperature of the emitter. For simplicity of demonstration, this modified figure of merit assumes a unity view factor of the filter and also neglects the angular dependence of thermal emission and transmission, though these effects could be incorporated.

Inspired by the work of Soljacic and co-workers [27], we optimize the geometry of 90-layer filters consisting of $\mathrm{Ta}_{2} \mathrm{O}_{5} / \mathrm{SiO}_{2}$ for three different thermal emitters: one emitter
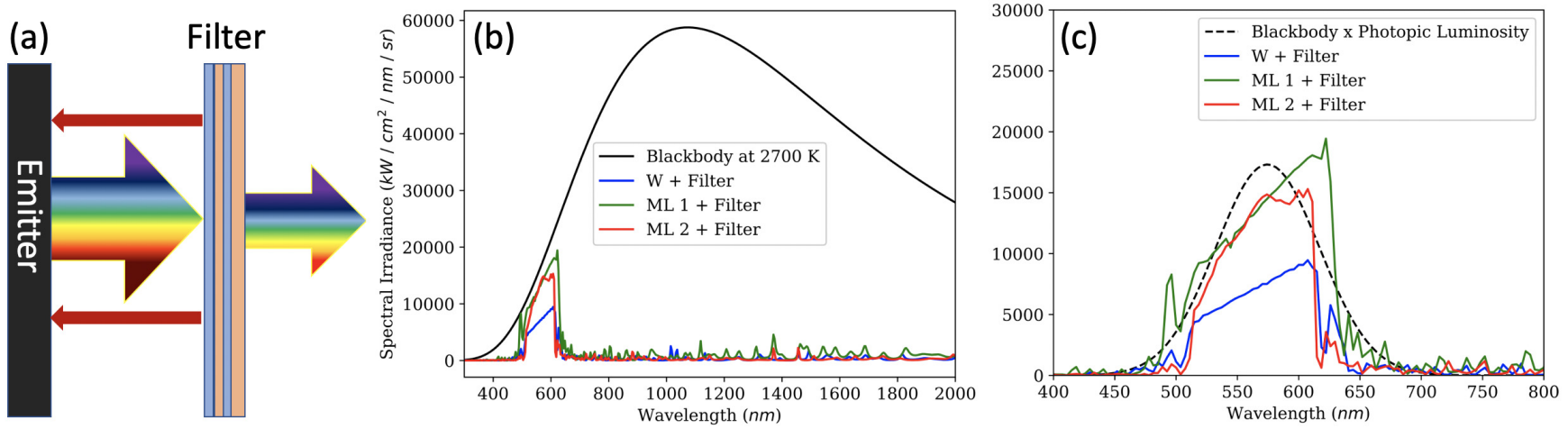

FIG. 2. Optimization of multilayer filters for incandescent sources at $2700 \mathrm{~K}$. Panel (a) illustrates the general structure where a thermal emitter (which may or may not be engineered itself) emits visible and IR radiation, and a filter between the emitter and the observer transmits visible radiation to the observer and reflects IR radiation back to the emitter. (b) Thermal radiation of three different thermal emitters filtered by multilayers optimized specifically for the emitter compared to blackbody emission. (c) Filtered thermal radiation of three different radiation sources compared to the photopic luminosity function multiplied by the blackbody spectrum at $2700 \mathrm{~K}$, which can be taken to be the ideal thermal emission at that temperature. 
TABLE II. Comparison of luminous efficiencies and visible power outputs of three different emitter-filter pairs. Each filter consists of a 90-layer stack of alternating $\mathrm{Ta}_{2} \mathrm{O}_{5}-\mathrm{SiO}_{2}$ specifically optimized for the luminous efficiency when paired with its respective emitter (see Appendix B for more details of the emitters and filters). Additionally, the mean time is reported for each geometry update using the L-BFGS-B algorithm utilizing analytic gradients compared to numeric gradients demonstrating the tremendous advantage of using analytical gradients for structures with many degrees of freedom. The luminous efficiency and visible power output of the lone emitters, as well as the emitters paired with an unoptimized 90-layer filter, is also reported for comparison, showing that optimized filters can have a significant impact on luminous efficiency, albeit with non-negligible reduction in brightness. Timings were performed on a workstation running Fedora 22 on a single 1.9-GHz Intel Xeon Processor.

\begin{tabular}{|c|c|c|c|c|}
\hline Structure & $\begin{array}{l}\eta_{\mathrm{Lum}}^{\mathrm{opt}} \\
(\%)\end{array}$ & $\begin{array}{c}P_{\text {vis }} \\
\left(\mathrm{W} / \mathrm{cm}^{2}\right)\end{array}$ & $\begin{array}{c}\left\langle t_{\Delta \mathbf{s}}^{\text {Analytic }}\right\rangle \\
(\mathrm{s})\end{array}$ & $\begin{array}{c}\left\langle t_{\Delta \mathrm{s}}^{\text {Numeric }}\right\rangle \\
\text { (s) }\end{array}$ \\
\hline $\mathrm{W}+F_{\mathrm{opt}}$ & 39.8 & 2.81 & 69.3 & 457.9 \\
\hline $\mathrm{ML} 1+F_{\mathrm{opt}}$ & 31.2 & 5.97 & 74.9 & 466.8 \\
\hline $\mathrm{ML} 2+F_{\mathrm{opt}}$ & 51.2 & 4.11 & 67.2 & 515.5 \\
\hline $\mathrm{W}+F_{i}$ & 22.6 & 5.65 & $\mathrm{~N} / \mathrm{A}$ & $\mathrm{N} / \mathrm{A}$ \\
\hline $\mathrm{ML} 1+F_{i}$ & 18.4 & 10.8 & $\mathrm{~N} / \mathrm{A}$ & $\mathrm{N} / \mathrm{A}$ \\
\hline $\mathrm{ML} 2+F_{i}$ & 35.1 & 7.12 & $\mathrm{~N} / \mathrm{A}$ & $\mathrm{N} / \mathrm{A}$ \\
\hline $\mathrm{W}$ & 3.8 & 7.26 & $\mathrm{~N} / \mathrm{A}$ & $\mathrm{N} / \mathrm{A}$ \\
\hline ML1 & 3.2 & 13.9 & $\mathrm{~N} / \mathrm{A}$ & $\mathrm{N} / \mathrm{A}$ \\
\hline ML2 & 6.2 & 8.92 & $\mathrm{~N} / \mathrm{A}$ & $\mathrm{N} / \mathrm{A}$ \\
\hline
\end{tabular}

made of pure tungsten (denoted W in Table II and Fig. 2), and two engineered multilayer emitters (denoted ML1 and ML2 in Table II and Fig. 2). ML1 is meant to model a realizable multilayer consisting of a tungsten base coated with an alloy of titanium nitride and aluminum nitride and an aluminum oxide top layer, while ML2 is a more idealized multilayer again with a tungsten base separated by a dielectric reflector from a selective absorber, which is modeled as a Lorentz oscillator. The specific geometries and material parameters of ML1 and ML2, along with all filters, can be found in the Appendixes, and Jupyter notebooks that set up optimizations and simulate these stacks can be found in the WPTherml repository $[37,38]$. These optimizations are performed using the L-BFGS-B algorithm driven by analytic gradients of Eq. (22) assuming a fixed temperature emitter of $2700 \mathrm{~K}$. This temperature is specifically chosen because standard $60-\mathrm{W}$ incandescent bulbs are typically reported to have a color temperature of $2700 \mathrm{~K}$, and because our engineered multilayered emitters have visible emissivity approaching unity (the blackbody limit), their thermodynamic temperature will be close to their color temperature. We initiate the filter geometries for each emitter as the precise geometry of a 90 -layer $\mathrm{Ta}_{2} \mathrm{O}_{5} / \mathrm{SiO}_{2}$ filter reported by Soljacic and co-workers [27] that was optimized for a tungsten emitter at $T=3000 \mathrm{~K}$. Note that it would not be expected that this structure should be a local optimum even for tungsten at $T=2700 \mathrm{~K}$ because of the impact that temperature has on the thermal radiation, and indeed we see that the filters can be optimized further for all three emitters; interested readers are encouraged to experiment with the impact of temperature on the optimizations of such filters using the previously referenced Jupyter notebook [37]. Because we are optimizing over the filters only, and it is the transmissivity of the filters that directly impacts $\eta_{\text {lum }}$, we compute analytic gradients of the transmissivity $\left(\frac{\partial T_{F}(\lambda)}{\partial s_{i}}\right)$ to drive the L-BFGS$\mathrm{B}$ updates. In addition to the luminous efficiency, which is directly optimized for, we report the visible power output computed as $P_{\text {vis }}=\pi \int_{\lambda}^{\lambda_{\max }} B\left(\lambda, T_{e}\right) \epsilon_{e}(\lambda) T_{F}(\lambda) d \lambda$, where $\lambda_{\min }=450 \mathrm{~nm}$ and $\lambda_{\max }=700 \mathrm{~nm}$.

The base case of a bare tungsten emitter has a luminous efficiency of $3.8 \%$ at $2700 \mathrm{~K}$; the first engineered multilayer (ML1) has a similar luminous efficiency but is expected to produce brighter light owing to a higher emissivity across the visible spectrum that yields roughly twice the visible power output of bare tungsten (13.9 compared to $7.2 \mathrm{~W} / \mathrm{cm}^{2}$; see Table II and Fig. 2). On the other hand, the second engineered multilayer (ML2) has a luminous efficiency that is more than $50 \%$ higher than bare tungsten but with only slightly greater visible power output (see Table II). The use of an engineered multilayer filter has a profound impact on the performance of the incandescent source. Using the initial filter geometry taken from Ref. [27] increases the luminous efficiency by a factor of greater than 5.5 for all emitter structures. Further optimization of each filter for its specific emission source leads to even more dramatic improvements in the luminous efficiency, with values of $39.8 \%, 31.2 \%$, and $51.2 \%$ for the bare tungsten emitter, ML1, and ML2 emitters plus optimized filters, respectively (see Table II). Each analytic gradient driven optimization took roughly $24 \mathrm{~h}$ on a single processor. We compare the time-per-geometry update averaged over the first 100 updates of the geometries for each filter using analytic gradients to the same updates using numerical gradients, finding that analytic gradient optimization steps are between 6.5 and 7.7 times faster than optimization steps utilizing numeric gradients (see Table II). Table II summarizes the luminous efficiency and visible power output of each lone emitter, each emitter paired with an unoptimized emitter, and each emitter paired with an optimized filter for ease of comparison; we note that timing data are only applicable for the emitters paired with optimized filters, which appear in the first three rows of the table.

An interesting facet of optimizing selective filters for luminous efficiency is that it tends to compete with visible power output of the emitter-filter pair, as we see that all emitters with their optimized filters are roughly ten times higher in luminous efficiency as compared to their base cases, but have all had their visible power outputs reduced by more than a factor of 2. It may therefore be worthwhile to consider a multiobjective optimization approach for filters and/or emitters perhaps involving the luminous power output in addition to the luminous efficiency. Notice also that the filtered thermal emission tends to be low at the short- and long-wavelength tails of the photopic luminosity function, which may affect the aesthetics of the filtered light; therefore inclusion of objective that captures the color rendering index may be desirable as well for future work.

\section{B. Optimization of antireflective coating for Si PV cell}

As a second case, we consider optimization of antireflective coatings for silicon photovoltaic (PV) cells. Here, the $\mathrm{PV}$ conversion efficiency can be expressed as $\eta_{\mathrm{PV}}=\frac{J_{\mathrm{sc}} V_{\mathrm{oc}} \mathrm{FF}}{P_{\mathrm{in}}}$, where $J_{\mathrm{sc}}$ is the short circuit current, $V_{\mathrm{oc}}$ is the open circuit 
(a)

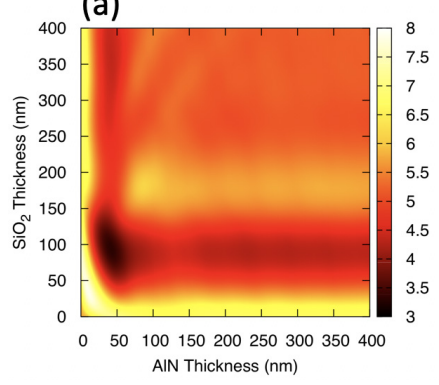

(b)

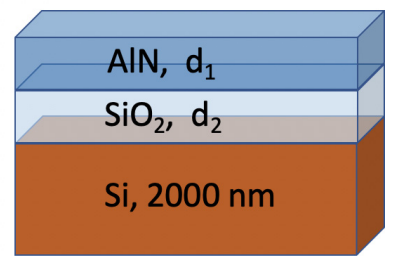

FIG. 3. PV Conversion efficiency of a $\mathrm{AlN} / \mathrm{SiO}_{2} / \mathrm{Si}$ stack where the (top) $\mathrm{AlN}$ and (middle) $\mathrm{SiO}_{2}$ layer thicknesses are adjusted and the (bottom) Si layer is fixed at $2000 \mathrm{~nm}$. There are multiple local optima even for this simple structure, illustrating the ruggedness of the efficiency surfaces of such structures. Within this search space, $\eta_{\mathrm{PV}}=7.87$ with $d_{1}=10.01 \mathrm{~nm}$ and $d_{2}=36.01 \mathrm{~nm}$.

voltage, FF is the fill factor, and $P_{\text {in }}$ is the total incident solar power. In this work, we take $V_{\text {oc }}$ and FF to have values of $0.706 \mathrm{mV}$ and 0.828 (unitless) in all cases (following Ref. [39]). The short circuit current can be computed as

$$
J_{\mathrm{sc}}=\int_{0}^{\infty} I_{\mathrm{AM} 1.5}(\lambda) \epsilon(\lambda) \operatorname{SP}(\lambda) d \lambda,
$$

where $I_{\mathrm{AM} 1.5}(\lambda)$ is the AM1.5 solar spectrum and $S P(\lambda)$ is the spectral response of monocrystalline silicon, which we take from Ref. [40], and $P_{\text {in }}$ can be computed as the integral over all wavelengths of the AM1.5 solar spectrum. We consider three different multilayers with $L=2,3$, and 4 layers of alternating $\mathrm{AlN} / \mathrm{SiO}_{2}$ atop $2-\mu \mathrm{m}$-thick silicon; in all cases $\mathrm{SiO}_{2}$ is the layer in direct contact with silicon (see Fig. 3 for an illustration of the $L=2$ system). In these stacks, only silicon is absorbing, which permits us to directly attribute the absorption of the multilayer stack as computed by the transfer-matrix method to the absorption within the active layer of the PV. The design of optical coatings for PV devices has been extensively pursued through computational optimization, albeit without the aid of analytic gradients (see Ref. [4] and references therein), and our purpose is primarily to illustrate the utility and flexibility afforded by our analytic gradient engine for accelerating global searches of rough landscapes.

We perform global optimizations of each multilayer coating by coupling a basin hopping algorithm $[35,36]$ to a L-BFGS-B algorithm that utilizes our analytic gradient engine. In this case, we assume only $J_{\text {sc }}$ [Eq. (23)] depends upon the multilayer thicknesses, and in particular, gradients of the absorptivity/emissivity $\left(\frac{\partial \epsilon(\lambda)}{\partial s_{i}}\right)$ are computed. We constrain the range of thicknesses for each layer to be within ranges that permit confirmation of the global optimum found by the BH algorithm by a brute force search (see Appendix $\mathrm{C}$ for details on these ranges). The figure of merit surface for the 2-layer case $\left(\eta_{\mathrm{PV}}\left(d_{1}, d_{2}\right)\right)$ is illustrated in Fig. 3, and it can be seen that even the 2-degree of freedom case yields a rugged landscape with multiple local extrema. Nevertheless, the BH algorithm coupled to the L-BFGS-B local optimization is capable of locating the global optimum in all cases. Furthermore, it is found that such optimizations driven by analytic gradients are roughly $2 \mathrm{x}$ faster than comparable optimizations driven by nu-
TABLE III. Comparison of efficiency of multiple optimizations performed within global searches for different $L$-layer antireflection coatings for a Si-based photovoltaic. In each case, 100 different local optimizations are performed with random initial geometries using analytic gradients, numeric gradients, or no gradients for the local optimization, respectively. The average time over these 100 different local optimizations is reported, along with the optimal conversion efficiency. Timings were performed on a workstation running Fedora 22 on a single 1.9-GHz Intel Xeon Processor. Note that bare Si has efficiency of $5.73 \%$.

\begin{tabular}{|c|c|c|c|c|c|}
\hline \multirow[t]{2}{*}{$L$} & \multicolumn{2}{|c|}{ Analytic } & \multicolumn{2}{|c|}{ Numeric } & \multirow{2}{*}{$\begin{array}{c}\text { Brute force } \\
\qquad \eta_{\mathrm{PV}}^{\mathrm{opt}}\end{array}$} \\
\hline & $\begin{array}{l}\eta_{\mathrm{PV}}^{\mathrm{opt}} \\
(\%)\end{array}$ & $\begin{array}{l}\text { Mean time } \\
\text { (s) }\end{array}$ & $\begin{array}{l}\eta_{\mathrm{PV}}^{\mathrm{opt}} \\
(\%)\end{array}$ & $\begin{array}{c}\text { Mean time } \\
\text { (s) }\end{array}$ & \\
\hline 2 & 7.87 & 13.8 & 7.87 & 18.2 & 7.87 \\
\hline 3 & 8.33 & 17.6 & 8.33 & 27.8 & 8.33 \\
\hline 4 & 8.61 & 38.4 & 8.61 & 75.8 & 8.61 \\
\hline
\end{tabular}

merical gradients (see Table III). We note that efficiency gain is more modest in this example because the number of degrees of freedom is relatively small, and we have shown previously the advantage of using analytical gradients becomes more apparent as the number of degrees of freedom grows. Geometries for the optimal structures are given in Appendix D, and Jupyter notebooks that set up these optimizations and simulate these structures can be found in the WPTherml repository [41,42]; optimized absorptivities are shown in Fig. 4.

\section{Optimization of an optical cavity for enhanced light harvesting}

As a final example, we consider the optimization of multilayered optical cavities for enhancing light absorption by a $J$-aggregate film. A suitably designed optical cavity can dramatically enhance the resonant interaction of light within a thin-film absorber layer, as discussed within the context of $J$-aggregates deposited or embedded in thin films [43,44], or

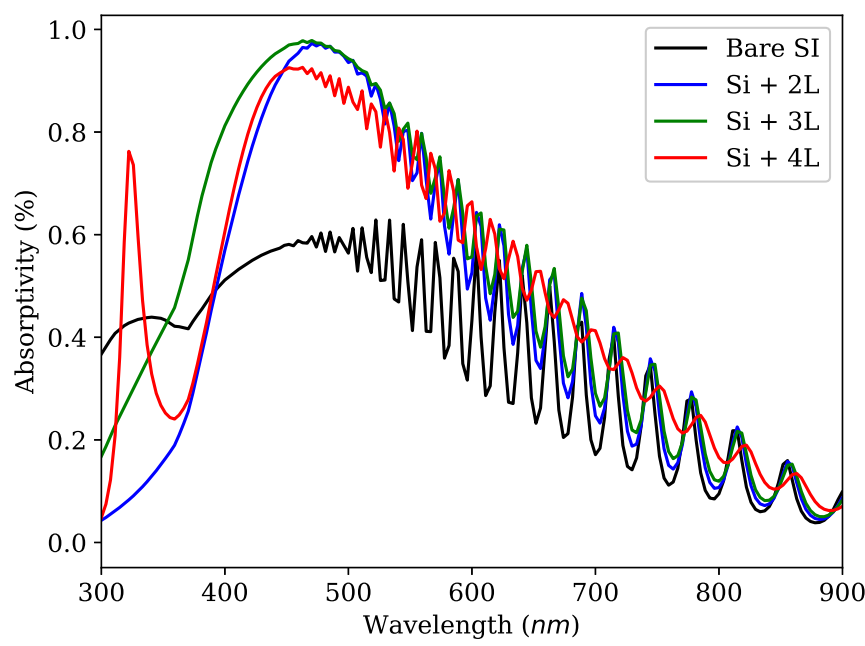

FIG. 4. Comparison of absorbance spectra of $2-\mu \mathrm{m}$ silicon with three different optimized multilayer coatings to the absorbance of bare $2-\mu \mathrm{m}$ silicon. 
(a)

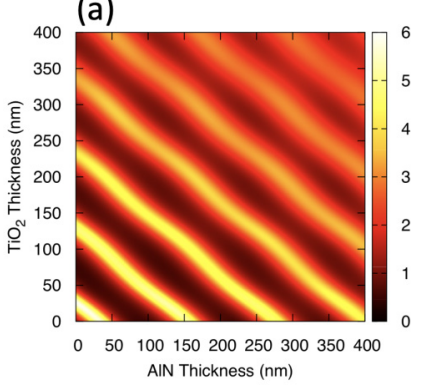

(b)

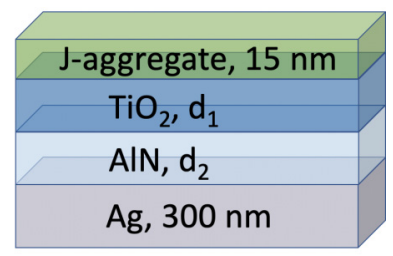

FIG. 5. (a) Absorption enhancement of a $J$-aggregate layer coupled to a multilayer reflector composed of $\mathrm{TiO}_{2} / \mathrm{AlN}$ backed by an $\mathrm{Ag}$ mirror as a function of the $\mathrm{AlN}$ and $\mathrm{TiO}_{2}$ thickness. A schematic of this structure is illustrated in (b).

with other weak absorbing layers $[14,45]$. Here we model the $J$-aggregate layer as a 15 -nm film with wavelength-dependent complex refractive index derived from the work of Wang [44], who considered cyanine-based $J$-aggregate dispersed in polyvinyl alcohol. We assume in all cases this $J$-aggregate layer exists as the top-most layer of a multilayer. Such a "freestanding" $J$-aggregate layer is found to absorb approximately $5 \%$ of incident light at the absorption resonance wavelength of $\lambda=580 \mathrm{~nm}$. By backing such a layer with an optical cavity that serves as a strong resonant reflector around $\lambda=580 \mathrm{~nm}$, the absorption of light into the $J$-aggregate layer can be enhanced. Such a strategy was shown by Akselrod et al. to ultimately enhance the fluorescence resonance energy-transfer efficiency from a similar $J$-aggregate donor to a laser dye acceptor [43]. Wang has also discussed the strong coupling of $J$-aggregate layers to high-finesse optical cavities to create hybrid light-matter states [44].

Here we consider simple optical cavities consisting of alternating $\mathrm{TiO}_{2}$ and AlN layers, where the AlN layer is chosen to be in contact with the silver back mirror. We seek simply to optimize the geometry of simple $L=2,3$, and 4 cavities for the enhancement of absorption of the composite structure relative to the free-standing $J$-aggregate film; the $L=2$ case is illustrated in Fig. 5. We define a figure of merit that quantifies the enhancement in absorption that results from the multilayer nanostructure relative to the base case of the 15 -nm $J$-aggregate film:

$$
E_{F}=\frac{\int_{\lambda_{\min }}^{\lambda_{\max }} \epsilon_{\mathrm{ML}}(\lambda) \epsilon_{J \mathrm{agg}}(\lambda) d \lambda}{\int_{\lambda_{\min }}^{\lambda_{\max }} \epsilon_{J \mathrm{ggg}}^{2}(\lambda) d \lambda},
$$

where $\epsilon_{\text {Jagg }}(\lambda)$ and $\epsilon_{\mathrm{ML}}(\lambda)$ denote the emissivity/absorptivity of the lone $J$-aggregate film and the multilayer toped with the $J$-aggregate film, respectively. The product between $\epsilon_{\mathrm{ML}}$ and $\epsilon_{J \mathrm{agg}}$ in the numerator of this figure of merit favors absorptivity/emissivity profiles that match the spectral shape of the lone $J$-aggregate absorption, and so tends to favor structures which directly enhance the absorption of the $J$ aggregate layer itself rather than structures which simply have high absorptivity in other spectral regions. The square of $\epsilon_{J \text { agg }}$ in the denominator normalizes the figure of merit so that if a multilayer structure has neither a positive nor a negative impact on $J$-aggregate absorption, the enhancement factor $E_{F}$ will have the value 1 . Because we fix the thickness of the lone
TABLE IV. Optimization of absorption enhancement of a 15-nm $J$-aggregate layer through the optimization of multilayer cavity separating the $J$-aggregate layer from a silver back mirror. In this case, increasing numbers of layers $L$ in the cavity beyond 2 does not improve the absorption enhancement; this result is found by both a basin hopping algorithm and a brute force search. The average time over 30 different local optimizations performed within the basin hopping using analytic and numeric gradients is reported along with the maximum enhancement found by the optimizations. Timings were performed on a workstation running Fedora 22 on a single 1.9-GHz Intel Xeon Processor.

\begin{tabular}{|c|c|c|c|c|c|}
\hline \multirow[t]{2}{*}{$L$} & \multicolumn{2}{|r|}{ Analytic } & \multicolumn{2}{|c|}{ Numeric } & \multirow{2}{*}{$\begin{array}{c}\text { Brute force } \\
\qquad E_{\mathrm{F}}^{\mathrm{opt}}\end{array}$} \\
\hline & $E_{\mathrm{F}}^{\mathrm{opt}}$ & Mean time (s) & $E_{\mathrm{F}}^{\mathrm{opt}}$ & Mean time (s) & \\
\hline 2 & 5.86 & 10.46 & 5.86 & 18.60 & 5.86 \\
\hline 3 & 5.86 & 18.09 & 5.86 & 43.32 & 5.86 \\
\hline 4 & 5.86 & 28.92 & 5.86 & 78.79 & 5.86 \\
\hline
\end{tabular}

$J$-aggregate film to be $15 \mathrm{~nm}$ and vary only the thicknesses of the multilayer coupled to this film, only $\epsilon_{\mathrm{ML}}(\lambda)$ has nonzero gradient elements associated with it. Therefore, optimizations of these multilayer structures is driven by gradients of the form

$$
\frac{\partial E_{F}}{\partial s_{i}}=\frac{\int_{\lambda_{\min }}^{\lambda_{\max }} \frac{\partial \epsilon_{\mathrm{ML}}(\lambda)}{\partial s_{i}} \epsilon_{J \mathrm{agg}}(\lambda) d \lambda}{\int_{\lambda_{\min }}^{\lambda_{\max }} \epsilon_{J \mathrm{agg}}^{2}(\lambda) d \lambda} .
$$

In our optimizations, we vary only the thickness of the dielectric layers so the gradient elements in Eq. (25) are only computed with respect to the thickness of the dielectric layers. We again consider a series of increasing dielectric layers from 2 to 4 so that global optimality can be confirmed by brute force search. However, unlike the PV coatings, we find no advantage for adding additional layers beyond the two dielectric layer case illustrated explicitly in Fig. 5. Nevertheless, we again see that analytic gradients provide a more expeditious route to optimizing the structure with the efficiency advantage becoming more dramatic as the number of degrees of freedom increases. As shown in Table IV, the mean optimization time is about $8 \mathrm{~s}$ faster using analytic gradients compared to numeric gradients for the $L=2$ case and about $50 \mathrm{~s}$ faster for the $L=4$ case. An example Jupyter notebook that performs these optimizations can be found in the WPTherml repository [46], and details of the optimal structures and plots of their absorptivity spectra can be found in the Appendix D.

\section{CONCLUSION}

We have presented a methodology for accelerating the optimization and virtual design of multilayer nanostructures for a variety of photonic and thermal radiation applications. This methodology is based upon a simple theoretical formulation of analytical gradients of the transfer-matrix equations with respect to geometric parameters of the multilayers. A practical implementation of this methodology is provided in open-source software package WPTherml [28], and we have leveraged this implementation to demonstrate the usefulness of this methodology to designing high efficiency incandescent structures, high-performance antireflection coatings for 
PV cells, and optical cavities for enhanced light harvesting by aggregates of organic chromophores in thin film. The methodology presented here will provide an important tool for the design and optimization of multilayer nanomaterials for a myriad of applications where tailored optical and thermal radiation properties are paramount.

\section{ACKNOWLEDGMENTS}

Acknowledgment is made to the donors of The American Chemical Society Petroleum Research Fund for partial support of this research. A.M. and D.W. acknowledge the GS-LSAMP program of the National Science Foundation for support. J.J.F. and J.F.V. acknowledge the College of Science and Health at William Paterson University for partial support of this research. J.J.F. acknowledges the ART program at William Paterson University for partial support of this research.

\section{APPENDIX A: EXPLICIT EXPRESSIONS OF D AND P MATRICES}

The transfer matrix is a product of matrices for each layer in the multilayer stack:

$$
\left(\begin{array}{ll}
M_{11} & M_{12} \\
M_{21} & M_{22}
\end{array}\right)=\mathbf{D}_{1}^{-1}\left(\prod_{l=2}^{L-1} \mathbf{D}_{l} \mathbf{P}_{l} \mathbf{D}_{l}^{-1}\right) \mathbf{D}_{L} .
$$

The terminal layers, layer 1 and layer $L$, are taken to be semi-infinite, and the central layers (layer 2, $L-1$ ) have finite thickness. Furthermore, the terminal layers have real refractive indices, while the central layers may have complex refractive indices. The $\mathbf{P}$ matrix is defined for each of the central layers:

$$
\mathbf{P}_{l}=\left(\begin{array}{cc}
\exp \left(-i k_{z, l} d_{l}\right) & 0 \\
0 & \exp \left(i k_{z, l} d_{l}\right)
\end{array}\right)
$$

where

$$
k_{z, l}=\sqrt{\left(n_{l} \frac{\omega}{c}\right)^{2}-\left(n_{l} \sin \left(\theta_{1}\right) \frac{\omega}{c}\right)^{2}},
$$

$d_{l}$ and $n_{l}$ is the thickness and refractive index of layer $l$, respectively, $\omega$ is the frequency of light, $\theta_{1}$ is the angle of incidence upon the multilayer structure, and $c$ is the vacuum speed of light. We note that $n_{l}$ often depends upon $\omega$ for materials of interest. The $\mathbf{D}$ matrices are defined for all layers, and depend upon the incident polarization of light. For $p$ polarized light

$$
\mathbf{D}_{l}=\left(\begin{array}{cc}
\cos \left(\theta_{1}\right) & \cos \left(\theta_{l}\right) \\
n_{l} & -n_{l}
\end{array}\right)
$$

and for $s$-polarized light

$$
\mathbf{D}_{l}=\left(\begin{array}{cc}
1 & 1 \\
n_{l} \cos \left(\theta_{l}\right) & -n_{l} \cos \left(\theta_{l}\right)
\end{array}\right)
$$

where $\theta_{l}$ is the refraction angle into the $l$ th layer that satisfies Snell's law [29]: $\theta_{l}=\arcsin \left[n_{1} / n_{l} \sin \left(\theta_{1}\right)\right]$. The matrices $\mathbf{D}_{l}^{-1}$ simply denote the inverse of the matrices $\mathbf{D}_{l}$.

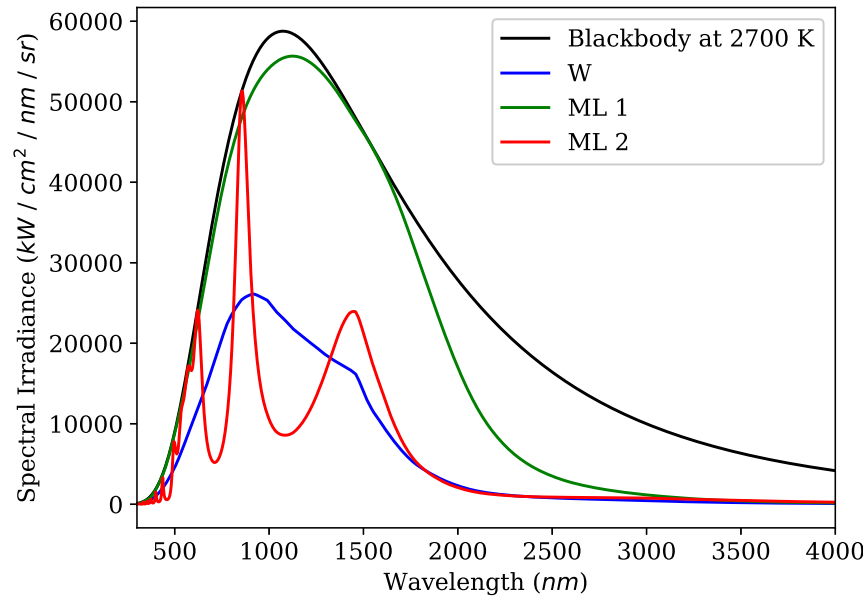

FIG. 6. Thermal emission of unfiltered emitters compared to blackbody spectrum at $2700 \mathrm{~K}$.

\section{APPENDIX B: DETAILS OF INCANDESCENT EMITTER/FILTER STRUCTURES}

The details of the 90-layer filters reported in the main text are provided in Tables V and VI. The emitter denoted ML1 consists of a 60-nm alumina top layer, a 90-nm central layer of $17 \%$-by-volume alloy of titanium nitride in aluminum nitride, and a terminal (bottom) tungsten layer that is optically thick. The Bruggeman effective-medium approximation is used to model the titanium nitride/aluminum nitride alloy [47]. The emitter denoted ML2 consists of 31-nm theoretical material modelled as a Lorentz oscillator [48], 85-nm aluminum nitride, $125 \mathrm{~nm}$ silicon dioxide, $174 \mathrm{~nm}$ aluminum nitride, $173 \mathrm{~nm}$ silicon dioxide, with a terminal optically thick tungsten layer. The refractive index for the Lorentz oscillator layer is defined as

$$
n_{l}=\sqrt{1+\frac{\omega_{p}^{2}}{\omega_{0}^{2}-\omega^{2}-i \omega \gamma}},
$$

where $\omega_{p}=\frac{2 \pi c}{1000 \mathrm{~nm}}, \gamma=\frac{2 \pi c}{7500 \mathrm{~nm}}$, and $\omega_{0}=\frac{2 \pi c}{550 \mathrm{~nm}}$. The unfiltered thermal emission spectra of the incandescent emitters are plotted in Fig. 6.

\section{APPENDIX C: DETAILS OF ANTIREFLECTIVE COATING STRUCTURES}

We perform optimizations over two-, three-, and four-layer antireflective coatings for $2-\mu \mathrm{m}$ silicon solar cells. The twolayer coating along with the silicon base is as follows: $10 \mathrm{~nm}$ aluminum nitride, $35 \mathrm{~nm}$ silicon dioxide, $2000 \mathrm{~nm}$ silicon. The three-layer coating along with the silicon base is as follows: $49 \mathrm{~nm}$ silicon dioxide, $11 \mathrm{~nm}$ aluminum nitride, $20 \mathrm{~nm}$ silicon dioxide, $2000 \mathrm{~nm}$ silicon. The four-layer coating along with the silicon base is as follows: $4 \mathrm{~nm}$ aluminum nitride, $49 \mathrm{~nm}$ silicon dioxide, $38 \mathrm{~nm}$ aluminum nitride, $10 \mathrm{~nm}$ silicon dioxide, $2000 \mathrm{~nm}$ silicon. In the two-layer optimization, we bound the thicknesses of each of the antireflective layers to be between 0.01 and $400.01 \mathrm{~nm}$ and we confirm the location 
TABLE V. First 45 layers of 90-layer filters used in conjunction with three different incandescent light sources. The layer thicknesses in column $2\left(d_{\text {init }}\right)$ represent the initial structure, taken from Ref. [27], and used as the starting point for all optimizations. The layer thicknesses in column $3\left(d_{\mathrm{opt}}^{M L 1}\right)$ resulted from the optimization for the emitter termed $M L 1$, layer thicknesses in column $4\left(d_{\mathrm{opt}}^{M L 2}\right)$ resulted from the optimization for the emitter termed $M L 2$, and layer thicknesses in column $5\left(d_{\mathrm{opt}}^{W}\right)$ resulted from the optimization for a bare tungsten emitter.

\begin{tabular}{|c|c|c|c|c|}
\hline Material & $\begin{array}{c}d_{\text {init }} \\
(\mathrm{nm})\end{array}$ & $\begin{array}{l}d_{\mathrm{opt}}^{\mathrm{ML} 1} \\
(\mathrm{~nm})\end{array}$ & $\begin{array}{l}d_{\mathrm{opt}}^{\mathrm{ML} 2} \\
(\mathrm{~nm})\end{array}$ & $\begin{array}{c}d_{\mathrm{opt}}^{W} \\
(\mathrm{~nm}\end{array}$ \\
\hline $\mathrm{Ta}_{2} \mathrm{O}_{5}$ & 18 & 6 & 12 & 8 \\
\hline $\mathrm{SiO}_{2}$ & 47 & 57 & 19 & 44 \\
\hline $\mathrm{Ta}_{2} \mathrm{O}_{5}$ & 156 & 205 & 223 & 21 \\
\hline $\mathrm{SiO}_{2}$ & 212 & 186 & 126 & 16 \\
\hline $\mathrm{Ta}_{2} \mathrm{O}_{5}$ & 178 & 168 & 215 & 201 \\
\hline $\mathrm{SiO}_{2}$ & 23 & 88 & 1 & 1 \\
\hline $\mathrm{Ta}_{2} \mathrm{O}_{5}$ & 51 & 1 & 1 & 71 \\
\hline $\mathrm{SiO}_{2}$ & 224 & 273 & 336 & 203 \\
\hline $\mathrm{Ta}_{2} \mathrm{O}_{5}$ & 150 & 211 & 207 & 20 \\
\hline $\mathrm{SiO}_{2}$ & 205 & 175 & 169 & \\
\hline $\mathrm{Ta}_{2} \mathrm{O}_{5}$ & 258 & 218 & 233 & \\
\hline $\mathrm{SiO}_{2}$ & 187 & 203 & 211 & \\
\hline $\mathrm{Ta}_{2} \mathrm{O}_{5}$ & 243 & 254 & 245 & \\
\hline $\mathrm{SiO}_{2}$ & 190 & 167 & 158 & \\
\hline $\mathrm{Ta}_{2} \mathrm{O}_{5}$ & 226 & 202 & 232 & \\
\hline $\mathrm{SiO}_{2}$ & 215 & 167 & 225 & \\
\hline $\mathrm{Ta}_{2} \mathrm{O}_{5}$ & 153 & 215 & 145 & \\
\hline $\mathrm{SiO}_{2}$ & 227 & 320 & 301 & \\
\hline $\mathrm{Ta}_{2} \mathrm{O}_{5}$ & 154 & 113 & 138 & \\
\hline $\mathrm{SiO}_{2}$ & 226 & 317 & 283 & \\
\hline $\mathrm{Ta}_{2} \mathrm{O}_{5}$ & 152 & 152 & 147 & \\
\hline $\mathrm{SiO}_{2}$ & 245 & 203 & 183 & \\
\hline $\mathrm{Ta}_{2} \mathrm{O}_{5}$ & 24 & 71 & 1 & \\
\hline $\mathrm{SiO}_{2}$ & 229 & 175 & 249 & \\
\hline $\mathrm{Ta}_{2} \mathrm{O}_{5}$ & 263 & 227 & 294 & \\
\hline $\mathrm{SiO}_{2}$ & 190 & 187 & 201 & \\
\hline $\mathrm{Ta}_{2} \mathrm{O}_{5}$ & 257 & 264 & 233 & \\
\hline $\mathrm{SiO}_{2}$ & 200 & 201 & 209 & \\
\hline $\mathrm{Ta}_{2} \mathrm{O}_{5}$ & 260 & 247 & 266 & \\
\hline $\mathrm{SiO}_{2}$ & 224 & 168 & 149 & \\
\hline $\mathrm{Ta}_{2} \mathrm{O}_{5}$ & 27 & 67 & 72 & \\
\hline $\mathrm{SiO}_{2}$ & 229 & 172 & 185 & \\
\hline $\mathrm{Ta}_{2} \mathrm{O}_{5}$ & 154 & 208 & 189 & \\
\hline $\mathrm{SiO}_{2}$ & 219 & 174 & 218 & \\
\hline $\mathrm{Ta}_{2} \mathrm{O}_{5}$ & 274 & 248 & 256 & \\
\hline $\mathrm{SiO}_{2}$ & 198 & 200 & 168 & \\
\hline $\mathrm{Ta}_{2} \mathrm{O}_{5}$ & 405 & 405 & 405 & \\
\hline $\mathrm{SiO}_{2}$ & 211 & 207 & 197 & \\
\hline $\mathrm{Ta}_{2} \mathrm{O}_{5}$ & 166 & 176 & 182 & \\
\hline $\mathrm{SiO}_{2}$ & 233 & 197 & 168 & \\
\hline $\mathrm{Ta}_{2} \mathrm{O}_{5}$ & 47 & 96 & 104 & \\
\hline $\mathrm{SiO}_{2}$ & 66 & 56 & 83 & \\
\hline $\mathrm{Ta}_{2} \mathrm{O}_{5}$ & 17 & 3 & 1 & 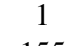 \\
\hline $\mathrm{SiO}_{2}$ & 125 & 136 & 143 & 155 \\
\hline $\mathrm{Ta}_{2} \mathrm{O}_{5}$ & 153 & 156 & 130 & 20 \\
\hline
\end{tabular}

of global optimum by a brute force search between these bounds with a resolution of $1 \mathrm{~nm}$ [Fig. 3(a)]. For the threeand four-layer cases, we bound the layers to be between 0.01
TABLE VI. Second 45 layers of 90-layer filters used in conjunction with three different incandescent light sources. The layer thicknesses in column $2\left(d_{\text {init }}\right)$ represent the initial structure, taken from Ref. [27], and used as the starting point for all optimizations. The layer thicknesses in column $3\left(d_{\mathrm{opt}}^{\mathrm{ML} 1}\right)$ resulted from the optimization for the emitter termed ML1, layer thicknesses in column $4\left(d_{\mathrm{opt}}^{\mathrm{ML} 2}\right)$ resulted from the optimization for the emitter termed ML2, and layer thicknesses in column $5\left(d_{\mathrm{opt}}^{W}\right)$ resulted from the optimization for a bare tungsten emitter.

\begin{tabular}{|c|c|c|c|c|}
\hline Material & $\begin{array}{c}d_{\text {init }} \\
(\mathrm{nm})\end{array}$ & $\begin{array}{l}d_{\mathrm{opt}}^{\mathrm{ML} 1} \\
(\mathrm{~nm})\end{array}$ & $\begin{array}{c}d_{\mathrm{opt}}^{\mathrm{ML} 2} \\
(\mathrm{~nm})\end{array}$ & $\begin{array}{c}d_{\mathrm{opt}}^{W} \\
(\mathrm{~nm})\end{array}$ \\
\hline $\mathrm{SiO}_{2}$ & 237 & 232 & 301 & 175 \\
\hline $\mathrm{Ta}_{2} \mathrm{O}_{5}$ & 151 & 150 & 136 & 192 \\
\hline $\mathrm{SiO}_{2}$ & 225 & 227 & 224 & 157 \\
\hline $\mathrm{Ta}_{2} \mathrm{O}_{5}$ & 147 & 123 & 151 & 124 \\
\hline $\mathrm{SiO}_{2}$ & 193 & 147 & 221 & 208 \\
\hline $\mathrm{Ta}_{2} \mathrm{O}_{5}$ & 127 & 117 & 146 & 107 \\
\hline $\mathrm{SiO}_{2}$ & 214 & 188 & 176 & 196 \\
\hline $\mathrm{Ta}_{2} \mathrm{O}_{5}$ & 135 & 114 & 120 & 140 \\
\hline $\mathrm{SiO}_{2}$ & 173 & 137 & 159 & 136 \\
\hline $\mathrm{Ta}_{2} \mathrm{O}_{5}$ & 112 & 101 & 108 & 102 \\
\hline $\mathrm{SiO}_{2}$ & 165 & 126 & 141 & 140 \\
\hline $\mathrm{Ta}_{2} \mathrm{O}_{5}$ & 130 & 103 & 128 & 98 \\
\hline $\mathrm{SiO}_{2}$ & 223 & 160 & 211 & 201 \\
\hline $\mathrm{Ta}_{2} \mathrm{O}_{5}$ & 130 & 134 & 164 & 122 \\
\hline $\mathrm{SiO}_{2}$ & 163 & 169 & 175 & 132 \\
\hline $\mathrm{Ta}_{2} \mathrm{O}_{5}$ & 112 & 108 & 88 & 83 \\
\hline $\mathrm{SiO}_{2}$ & 164 & 145 & 121 & 126 \\
\hline $\mathrm{Ta}_{2} \mathrm{O}_{5}$ & 114 & 129 & 95 & 90 \\
\hline $\mathrm{SiO}_{2}$ & 167 & 54 & 171 & 119 \\
\hline $\mathrm{Ta}_{2} \mathrm{O}_{5}$ & 121 & 135 & 163 & 92 \\
\hline $\mathrm{SiO}_{2}$ & 378 & 332 & 379 & 338 \\
\hline $\mathrm{Ta}_{2} \mathrm{O}_{5}$ & 114 & 112 & 116 & 130 \\
\hline $\mathrm{SiO}_{2}$ & 160 & 142 & 142 & 168 \\
\hline $\mathrm{Ta}_{2} \mathrm{O}_{5}$ & 113 & 103 & 105 & 92 \\
\hline $\mathrm{SiO}_{2}$ & 174 & 185 & 139 & 155 \\
\hline $\mathrm{Ta}_{2} \mathrm{O}_{5}$ & 117 & 152 & 100 & 107 \\
\hline $\mathrm{SiO}_{2}$ & 211 & 245 & 166 & 180 \\
\hline $\mathrm{Ta}_{2} \mathrm{O}_{5}$ & 23 & 1 & 93 & 83 \\
\hline $\mathrm{SiO}_{2}$ & 221 & 141 & 190 & 167 \\
\hline $\mathrm{Ta}_{2} \mathrm{O}_{5}$ & 261 & 260 & 303 & 212 \\
\hline $\mathrm{SiO}_{2}$ & 399 & 369 & 394 & 355 \\
\hline $\mathrm{Ta}_{2} \mathrm{O}_{5}$ & 266 & 258 & 287 & 236 \\
\hline $\mathrm{SiO}_{2}$ & 390 & 370 & 376 & 373 \\
\hline $\mathrm{Ta}_{2} \mathrm{O}_{5}$ & 28 & 258 & 1 & 7 \\
\hline $\mathrm{SiO}_{2}$ & 18 & 370 & 1 & 1 \\
\hline $\mathrm{Ta}_{2} \mathrm{O}_{5}$ & 367 & 25 & 357 & 405 \\
\hline $\mathrm{SiO}_{2}$ & 198 & 4 & 165 & 195 \\
\hline $\mathrm{Ta}_{2} \mathrm{O}_{5}$ & 302 & 341 & 267 & 380 \\
\hline $\mathrm{SiO}_{2}$ & 28 & 176 & 1 & 1 \\
\hline $\mathrm{Ta}_{2} \mathrm{O}_{5}$ & 33 & 365 & 97 & 1 \\
\hline $\mathrm{SiO}_{2}$ & 426 & 359 & 377 & 362 \\
\hline $\mathrm{Ta}_{2} \mathrm{O}_{5}$ & 31 & 1 & 1 & 1 \\
\hline $\mathrm{SiO}_{2}$ & 15 & 1 & 1 & 1 \\
\hline $\mathrm{Ta}_{2} \mathrm{O}_{5}$ & 222 & 228 & 242 & 229 \\
\hline $\mathrm{SiO}_{2}$ & 96 & 72 & 77 & 73 \\
\hline
\end{tabular}

and $49.01 \mathrm{~nm}$ and we confirm the location of global optimum using a resolution of 1 and $2.5 \mathrm{~nm}$ for the three- and four-layer case, respectively. 


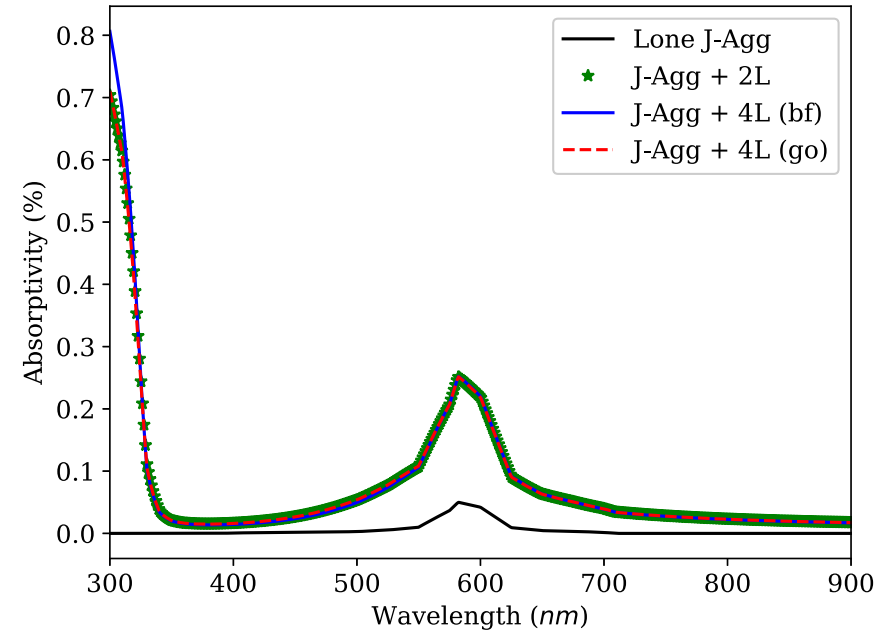

FIG. 7. Plot of absorptivity of $J$-aggregate thin film compared to $J$-aggregate thin films coupled to different optical cavities. The Lone $J$-Agg plot indicates the lone $15 \mathrm{~nm} J$-aggregate thin film, the $J$-Agg $+2 L$ is the structure that results from global optimization of the $J$-Agg + a two-layer optical cavity and silver backing mirror; this is identical within numerical precision to the optimal structure identified by a brute force search. The $J$-Agg $+4 L$ structures involve the four-layer optical cavities identified as optimal by a brute force search (bf) and by the basin hopping global optimization (go) routine, respectively. The absorptivity of these two structures is virtually identical in the visible region, and differ slightly in the UV absorbance due to different thickness of the $\mathrm{TiO}_{2}$ layers; note that the UV absorbance does not contribute to the objective function, hence these structures are not distinguished from each other by the optimizations.

\section{APPENDIX D: DETAILS OF THE OPTICAL CAVITIES FOR ABSORPTION ENHANCEMENT OF $J$-AGGREGATE LAYERS}

We perform optimizations over two-, three-, and four-layer optical cavities that separate a top-most 15 -nm $J$-aggregate film from an optically thick $(300 \mathrm{~nm})$ silver back mirror; the optimized spectra of all optimized structures are plotted in Fig. 7. Each optical cavity contained alternating layers of titanium dioxide $\left(\mathrm{TiO}_{2}\right)$ and aluminum nitride $(\mathrm{AlN})$ with AlN always in contact with the silver back mirror. The global optimum geometry for the two-layer case was determined to be $15 \mathrm{~nm} J$ aggregate, $8.01 \mathrm{~nm} \mathrm{TiO} 2,21.01 \mathrm{~nm} \mathrm{AlN,} 300$ $\mathrm{nm} \mathrm{Ag}$ by brute force and $15 \mathrm{~nm} J$ aggregate, $7.15 \mathrm{~nm} \mathrm{TiO}_{2}$, $21.59 \mathrm{~nm}$ AlN, $300 \mathrm{~nm} \mathrm{Ag}$ by the global optimization. The three-layer case was $15 \mathrm{~nm} J$ aggregate, $0.01 \mathrm{~nm}$ AlN, 6.01 $\mathrm{nm} \mathrm{TiO}_{2}, 22.51 \mathrm{~nm} \mathrm{AlN,} 300 \mathrm{~nm} \mathrm{Ag}$ by brute force and 15 $\mathrm{nm} J$ aggregate, $1.00 \mathrm{~nm} \mathrm{AlN,} 5.74 \mathrm{~nm} \mathrm{TiO}{ }_{2}, 21.59 \mathrm{~nm} \mathrm{AlN}$, and $300 \mathrm{~nm} \mathrm{Ag}$ by global optimization. The four-layer case was $15 \mathrm{~nm} J$ aggregate, $4.01 \mathrm{~nm} \mathrm{TiO} 2,0.01 \mathrm{~nm} \mathrm{AlN,} 0.01 \mathrm{~nm}$ $\mathrm{TiO}_{2}, 24.01 \mathrm{~nm}$ AlN, $300 \mathrm{~nm} \mathrm{Ag}$ by brute force and $15 \mathrm{~nm} J$ aggreagate, $6.14 \mathrm{~nm} \mathrm{TiO}_{2}, 1 \mathrm{~nm} \mathrm{AlN,} 1 \mathrm{~nm} \mathrm{TiO}$, and 20.59 AlN by global optimization.

In the two-layer optimization, we bound the thicknesses of each of the antireflective layers to be between 0.01 and $400.01 \mathrm{~nm}$ and we confirm the location of global optimum by a brute force search between these bounds with a resolution of $1 \mathrm{~nm}$ [Fig. 3(a)]. For the three- and four-layer cases, we bound

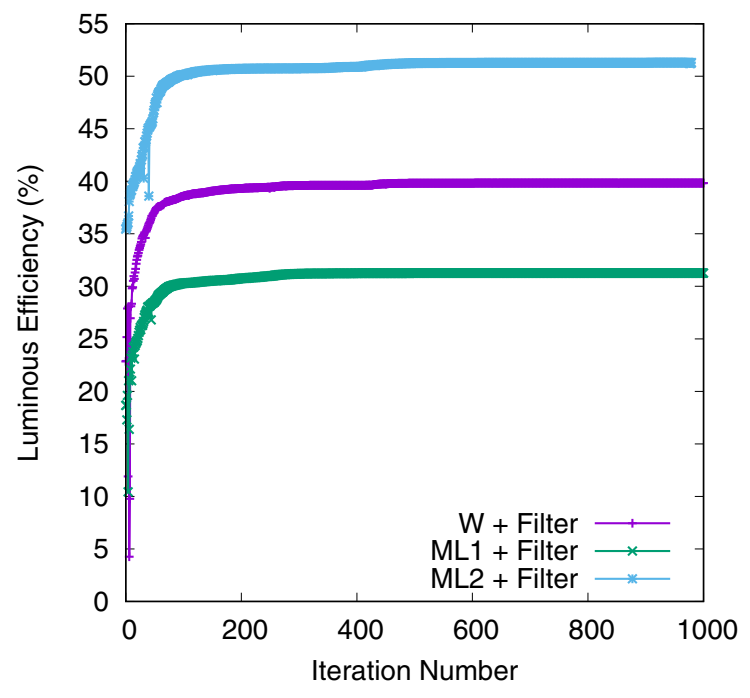

FIG. 8. Plot of luminous efficiency vs iteration number for the local optimization of the three distinct 90-layer incandescent filters discussed in Sec. III A.

the layers to be between 0.01 and $60.01 \mathrm{~nm}$ and we confirm the location of global optimum using a resolution of 1.5 and $2.0 \mathrm{~nm}$ for the three- and four-layer case, respectively.

\section{APPENDIX E: CONVERGENCE OF OPTIMIZATIONS}

Here we present plots of the figures of merit vs iteration number for each optimization case demonstrated in the main text. In Fig. 8 we show the luminous efficiency figure of merit vs iteration number for the three distinct 90-layer incandescent filters that were locally optimized using a bounded L-BFGS method.

For the solar coating and the $J$-aggregate spacer layers, global optimizations were performed that launched a large number of independent local L-BFGS-based optimizations. In Fig. 9, we plot the solar conversion efficiency figure of merit vs iteration number for the global optimization, and

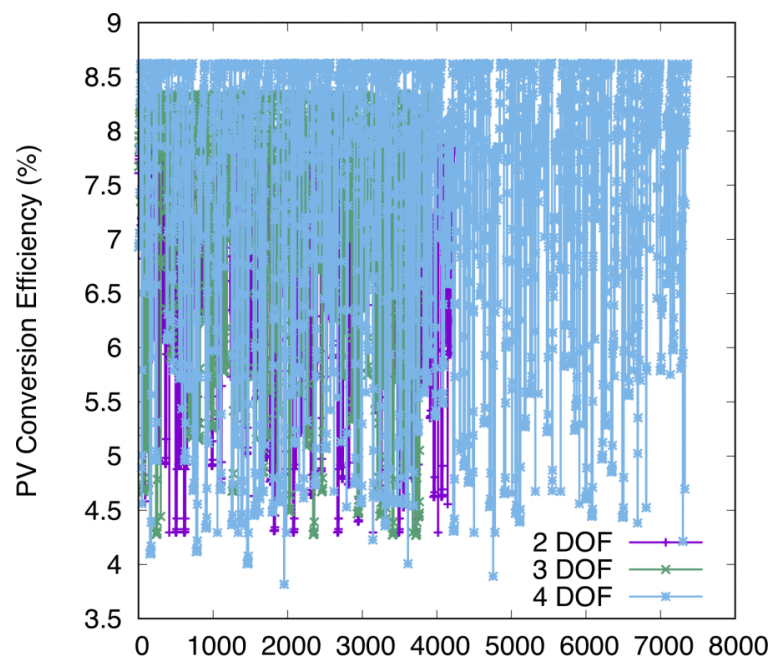

FIG. 9. Plot of conversion efficiency vs iteration number for the global optimization of the two-, three-, and four-layer antireflective coatings. 


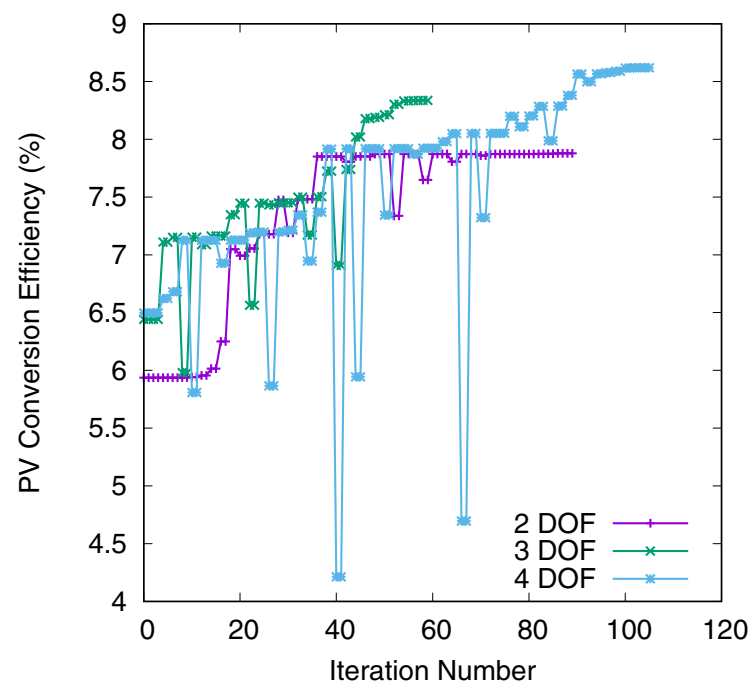

FIG. 10. Plot of conversion efficiency vs iteration number for local optimizations of the two-, three-, and four-layer antireflective coatings; these local optimizations represent independent subiterations of the global optimizations performed.

subsequently in Fig. 10 show this figure of merit vs iteration number for a single local optimization performed within the global optimization.

Finally, we plot the $J$-aggregate absorption enhancement figure of merit vs iteration number for the global optimization in Fig. 11, and subsequently in Fig. 12 show this figure of merit vs iteration number for a single local optimization performed within the global optimization.

\section{APPENDIX F: VALIDATION AGAINST FINITE-DIFFERENCE TIME-DOMAIN METHOD}

We provide a comparison of optimized emissivities determined by our method and emissivities of the same structures from a full-wave simulations; a commercial-grade simulator

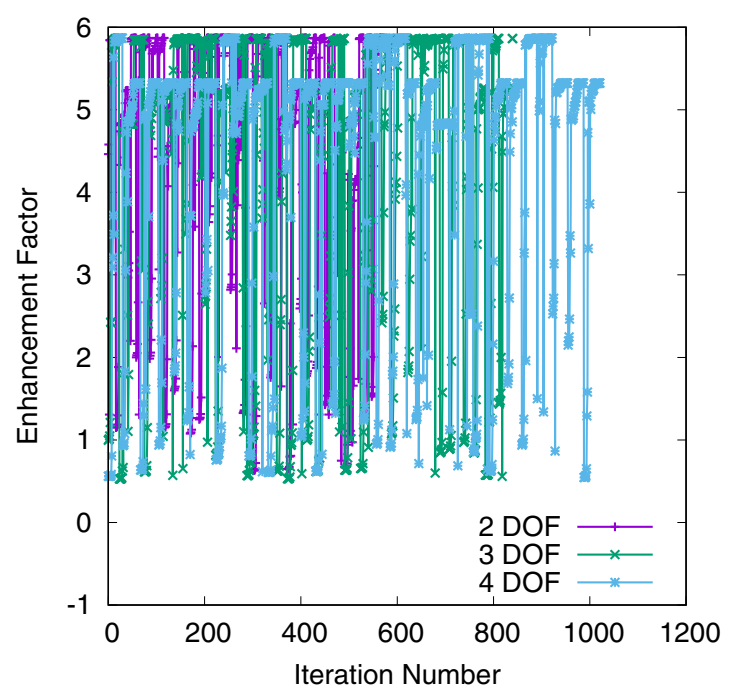

FIG. 11. Plot of conversion efficiency vs iteration number for the global optimization of the two-, three-, and four-layer antireflective coatings.

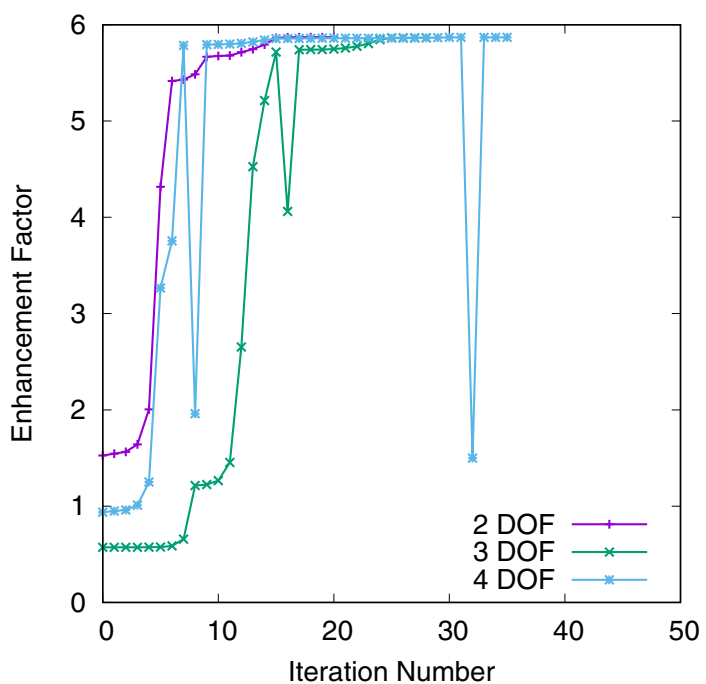

FIG. 12. Plot of conversion efficiency vs iteration number for local optimizations of the two-, three-, and four-layer antireflective coatings; these local optimizations represent independent subiterations of the global optimizations performed.

based on the finite-difference time-domain (FDTD) method was used to perform the full-wave calculations [49]. In the FDTD simulations, the multilayers were stratified along the $z$ axis and periodic along the $x$ and $y$ dimensions; the unit cell was $20 \mathrm{~nm} \times 20 \mathrm{~nm}$ in $x$ and $y$, and the total $z$ span was $4000 \mathrm{~nm}$ terminated with perfectly matched layers. A plane-wave source with a broadband spectrum spanning 300 $900 \mathrm{~nm}$ was incident along the positive $z$ axis and polarized along the $y$ axis. We consider two representative structures from the main text: the Bare Si structure and the $\mathrm{Si}+2 \mathrm{ML}$ structure from Sec. III B. The former structure is simply a 2000-nm silicon slab with air above and below, while the $\mathrm{Si}+2 \mathrm{ML}$ structure consists of $2000 \mathrm{~nm}$ silicon coated with $35.7 \mathrm{~nm}$ of $\mathrm{SiO}_{2}$ and $10.1 \mathrm{~nm}$ of $\mathrm{AlN}$ (see Ref. [42]). In the region containing the thin $\mathrm{SiO}_{2}$ and AlN layers, we use a fine mesh with a spacing of $0.4 \mathrm{~nm}$ along $z$ and $0.8 \mathrm{~nm}$ along

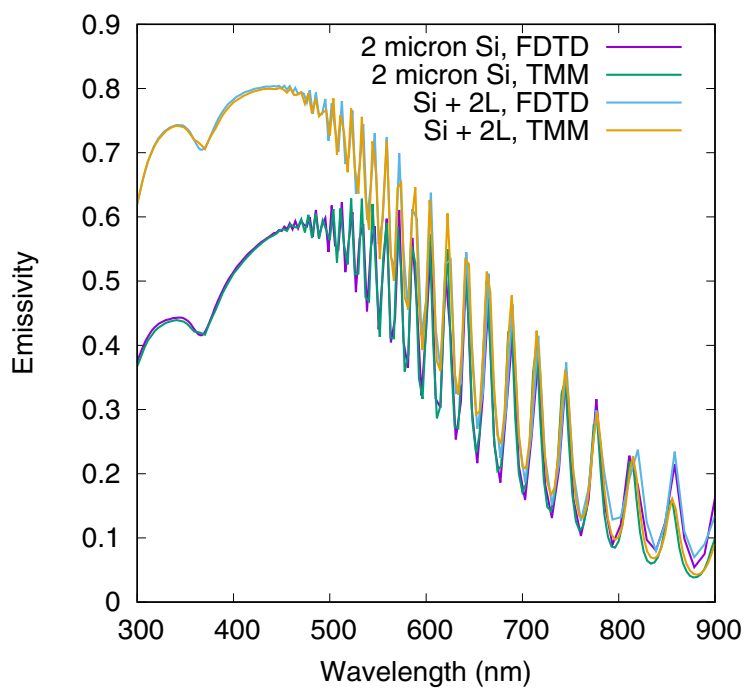

FIG. 13. Emissivity as predicted by the FDTD method and our method for two representative structures, $\mathrm{Si}$ and $\mathrm{Si}+2 L$. 
$x$ and $y$, and we apply an adaptive meshing scheme to the remaining simulation volume. Field flux monitors are placed behind the silicon slab and behind the injection source to infer the transmissivity and reflectivity of the multilayer, from which the emissivity can also be inferred. We also estimate the derivative of the emissivity with respect to the thickness of the AlN layer within the FDTD framework by applying a simple finite-difference approach whereby the FDTD emissivity is computed with a geometry of 10.1, 35.7, $2000 \mathrm{~nm}$ for AlN, $\mathrm{SiO}_{2}$, and $\mathrm{Si}$ and then 11.1, 35.7, and $2000 \mathrm{~nm}$ for $\mathrm{AlN}, \mathrm{SiO}_{2}$, and $\mathrm{Si}$. The numerically differentiated FDTD emissivities are then compared to the derivative of the emissivity with respect to the thickness of the AlN layer that can be analytically computed by our method as described in the main text (see Figs. 13 and 14). We make one adaptation for these comparisons, which is to model the $\mathrm{SiO}_{2}$ and AlN with static refractive indices of 1.45 and 1.859 , respectively. In the results presented in our main text, we use dispersive models for both materials that are described in Ref. [28]; the dispersive nature of AlN in particular is challenging for the FDTD method and obscures comparison. The close agreement between the emissivities and derivatives of the emissivities as computed by WPTherml and those computed by full-wave simulation support the claim that our method provides predictive power for

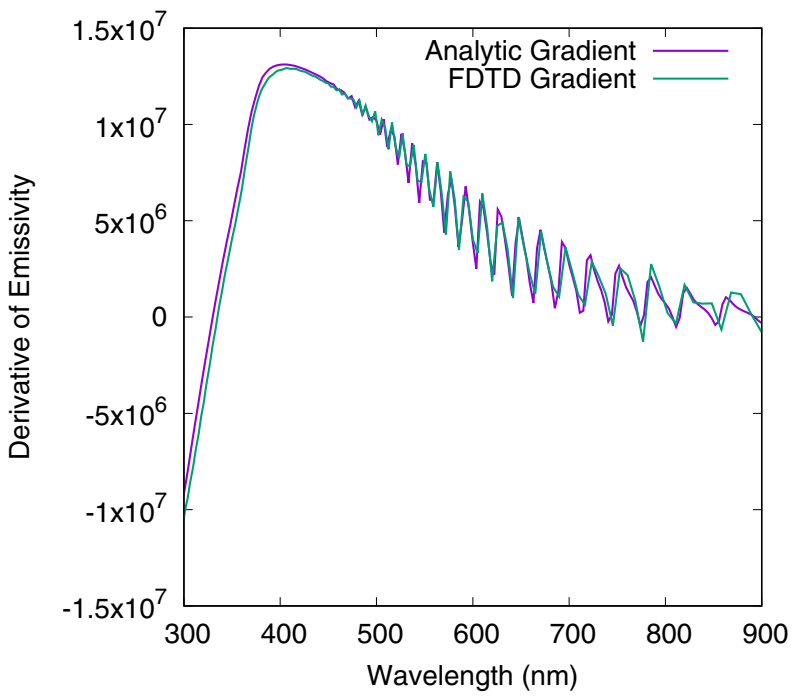

FIG. 14. Derivative of emissivity with respect to the thickness of the AlN layer in the $\mathrm{Si}+2 L$ structure predicted by the FDTD method and our method.

the optimization and design of structures whose performance derives from optical quantities like the emissivity.
[1] P. Ben-Abdallah and B. Ni, Single-defect Bragg stacks for highpower narrow-band thermal emission, J. Appl. Phys. 97, 104910 (2005).

[2] B. J. Lee and Z. M. Zhang, Design and fabrication of planar multilayer structures with coherent thermal emission characteristics, J. Appl. Phys. 100, 063529 (2006).

[3] E. Rephaeli and S. Fan, Absorber and emitter for solar thermophotovoltaic systems to achieve efficiency exceeding the schockley-queisser limit, Opt. Express 17, 15145 (2009).

[4] M. Ghebrebrhan, P. Bermel, Y. Avniel, J. D. Joannopoulos, and S. G. Johnson, Global optimization of silicon photovoltaic cell front coatings, Opt. Express 17, 7505 (2009).

[5] H. A. Atwater and A. Polman, Plasmonics for improved photovoltaic devices, Nat. Mater. 9, 205 (2010).

[6] E. Nefzaoui, J. Drevillon, and K. Joulain, Selective emitters design and optimization for thermophotovoltaic applications, J. Appl. Phys. 111, 084316 (2012).

[7] Y. Nam, Y. X. Yeng, A. Lenert, P. Bermel, I. Celanovic, M. Soljačić, and E. N. Wang, Solar thermophotovoltaic energy conversion systems with two-dimensional tantalum photonic crystal absorbers and emitters, Sol. Energy Mat. Sol. Cells 122, 287 (2014).

[8] V. Rinnerbauer, A. Lenert, D. M. Bierman, Y. X. Yeng, W. R. Chan, R. D. Geil, J. J. Senkevich, J. D. Joannopoulos, E. N. Wang, M. Soljačić, and I. Celanovic, Metallic photonic crystal absorber-emitter for efficient spectral control in hightemperature solar thermophotovoltaics, Adv. Energy Mater. 4, 1400334 (2014).

[9] A. Lenert, D. M. Bierman, Y. Nam, W. R. Chan, I. Celanovic, M. Soljačić, and E. N. Wang, A nanophotonic solar thermophotovoltaic device, Nat. Nanotechnol. 9, 126 (2014).
[10] Z. Zhou, E. Sakr, Y. Sun, and P. Bermel, Solar thermophotovoltaics: reshaping the solar spectrum, Nanophotonics 5, 1 (2016).

[11] D. Hayes, R. G. Hadt, J. D. Emery, A. A. Cordones, A. B. F. Martinson, M. L. Shelby, K. A. Fransted, P. D. Dahlberg, J. Hong, X. Zhang, Q. Kong, R. W. Schoenlein, and L. X. Chen, Electronic and nuclear contributions to time-resolved optical and $\mathrm{x}$-ray absorption spectra of hematite and insights into photoelectrochemical performance, Energy Environ. Sci. 9, 3754 (2016)

[12] N. Eldabagh, J. Czarnecki, and J. J. Foley IV, Nanophotonics with Novel Nanoparticles: New Phenomena and New Possibilities (Wiley, Hoboken, NJ, 2018), Chap. 6.

[13] M. C. Gupta, C. Ungaro, J. J. Foley IV, and S. K. Gray, Optical nanostructures design, fabrication, and applications for solar/thermal energy conversion, Solar Energy 165, 100 (2018).

[14] N. Jeon, J. J. Hernandez, D. Rosenmann, S. K. Gray, A. B. F. Martinson, and J. J. Foley IV, Pareto optimal spectrally selective emitters for thermophotovoltaics via weak absorber critical coupling, Adv. Energy Mater. 8, 1801035 (2018).

[15] A. Hima, N. Lakhdar, B. Benhaoua, A. Saadoune, I. Kemerchou, and F. Rogti, An optimized perovskite solar cell designs for high conversion efficiency, Superlattice Microstruct. 129, 240 (2019).

[16] Y. Hong, M. Otten, M. Min, S. K. Gray, and D. P. Nicholls, Periodic corrugations to increase efficiency of thermophotovoltaic emitting structures, Appl. Phys. Lett. 114, 053901 (2019).

[17] D. G. Baranov, Y. Xiao, I. A. Nechepurenko, A. Krasnok, A. Al, and M. A. Kats, Nanophotonic engineering of far-field thermal emitters, Nat. Mater. 18, 920 (2019). 
[18] E. Rephaeli, A. Raman, and S. Fan, Ultrabroadband photonic structures to achieve high-performance daytime radiative cooling, Nano Lett. 13, 1457 (2013).

[19] A. P. Raman, M. A. Anoma, L. Zhu, E. Rephaeli, and S. Fan, Passive radiative cooling below ambient air temperature under direct sunlight, Nature (London) 515, 540 (2014).

[20] Y. Zhai, Y. Ma, S. N. David, D. Zhao, R. Lou, G. Tan, R. Yang, and X. Yin, Scalable-manufactured randomized glass-polymer hybrid metamaterial for daytime radiative cooling, Science $\mathbf{3 5 5}$, 1062 (2017).

[21] J. Mandal, Y. Fu, A. C. Overvig, M. Jia, K. Sun, N. N. Shi, H. Zhou, X. Xiao, N. Yu, and Y. Yang, Hierarchically porous polymer coatings for highly efficient passive daytime radiative cooling, Science 362, 315 (2018).

[22] B. Bhatia, A. Leroy, Y. Shen, L. Zhao, M. Gianello, D. Li, T. Gu, J. Hu, M. Soljai, and E. N. Wang, Passive directional sub-ambient daytime radiative cooling, Nat. Commun. 9, 5001 (2018).

[23] Y. Zhu, D. Wang, C. Fang, P. H. P1, and Y.-H. Ye, A multilayer emitter close to ideal solar reflectance for efficient daytime radiative cooling, Polymers 11, 1203 (2019).

[24] F. J. Studer and D. A. Cusano, Titanium dioxide films as selective reflectors of the near-infrared, J. Opt. Soc. Am. 43, 522 (1953).

[25] B. E. Yoldas and T. O'Keefe, Deposition of optically transparent ir reflective coatings on glass, Appl. Opt. 23, 3638 (1984).

[26] Y.-S. Kim, S.-Y. Lin, A. S. P. Chang, J.-H. Lee, and K.-M. Ho, Analysis of photon recycling using metallic photonic crystal, J. Appl. Phys. 102, 063107 (2007).

[27] O. Ilic, P. Bermel, G. Chen, J. D. Joannopoulos, I. Celanovic, and M. Soljacic, Tailoring high-temperature radiation and the resurrection of the incandescent source, Nat. Nanotechnol. 11, 320 (2016)

[28] J. F. Varner, N. Eldabagh, D. Volta, R. S. Eldabagh, and J. J. Foley IV, WpTherml: A python package for the design of materials for harnessing heat, J. Open Res. Softw. 7, 28 (2019).

[29] P. Yeh, Optical Waves in Layered Media (Wiley, Hoboken, NJ, 2005).

[30] A. Mayer, L. Gaouyat, D. Nicolay, T. Carletti, and O. Deparis, Multi-objective genetic algorithm for the optimization of a flatplate solar thermal collector, Opt. Express 22, A1641 (2014).

[31] P. Bermel, M. Ghebrebrhan, W. Chan, Y. X. Yeng, M. Araghchini, R. Hamam, C. H. Marton, K. F. Jensen, M. Soljacic, J. D. Joannopoulos, S. G. Johnson, and I. Celanovic, Design and global optimization of high-efficiency thermophotovoltaic systems, Opt. Express 18, A314 (2010).

[32] J. Drevillon and P. Ben-Abdallah, Ab initio design of coherent thermal sources, J. Appl. Phys. 102, 114305 (2007).

[33] R. H. Byrd, P. Lu, and J. Nocedal, A limited memory algorithm for bound constrained optimization, SIAM J. Sci. Comput. 16, 1190 (1995).
[34] E. Maradzike, G. Gidofalvi, J. M. Turney, H. F. Schaefer III, and A. E. DePrince III, Analytic energy gradients for variational two-electron reduced-density-matrix-driven complete active space self-consistent field theory, J. Chem. Theory Comput. 13, 4113 (2017).

[35] D. J. Wales and J. P. K. Doye, Global optimization by basinhopping and the lowest energy structures of lennard-jones clusters containing up to 110 atoms, J. Phys. Chem. A 101, 5111 (1997).

[36] D. J. Wales and H. A. Scheraga, Global optimization of clusters, crystals, and biomolecules, Science 285, 1368 (1999).

[37] Optimization of multilayer filters for incandescent sources, https://github.com/FoleyLab/wptherml/blob/dev/example/ Incandescent_Opt.ipynb (2019), accessed: 2019-07-30.

[38] Plotting thermal emission of filtered incandescent sources, https://github.com/FoleyLab/wptherml/blob/dev/example/ Incandescent_Plot.ipynb (2019), accessed: 2019-07-30.

[39] L.-Z. Wang, Z.-Q. Zhou, T.-N. Zhang, X. Chen, and M. Lu, High fill factors of si solar cells achieved by using an inverse connection between mos and pn junctions, Nanoscale Res. Lett. 11, 453 (2016).

[40] A. P. Saffar and B. D. Barani, Thermal effects investigation on electrical properties of silicon solar cells treated by laser irradiation, Int. J. Renew. Energy Dev. 3, 184 (2014).

[41] Optimization of multilayer coatings for silicon solar cells, https://github.com/FoleyLab/wptherml/blob/dev/example/ PV_Opt.ipynb (2019), accessed: 2019-07-30.

[42] Plotting absorbance of silicon solar cells with and without optimized coatings, https://github.com/FoleyLab/wptherml/blob/ dev/example/PV_Plot.ipynb (2019), accessed: 2019-07-30.

[43] G. M. Akselrod, B. J. Walker, W. A. Tisdale, M. G. Bawendi, and V. Bulovic, Twenty-fold enhancement of molecular fluorescence by coupling to a j-aggregate critically coupled resonator, ACS Nano 6, 467 (2012).

[44] S. Wang, Strong light-molecule coupling: routes to new hybrid materials, Ph.D. thesis, Universite de Strasbourg, 2015.

[45] J. R. Piper and S. Fan, Total absorption in a graphene monolayer in the optical regime by critical coupling with a photonic crystal guided resonance, ACS Photon. 1, 347 (2014).

[46] Optimization of multilayer cavities for absorption enhancement, https://github.com/FoleyLab/wptherml/blob/dev/ example/J_aggregate_opt.ipynb (2019), accessed: 2019-09-07.

[47] O. Zhuromskyy, Applicability of effective medium approximations to modeling of mesocrystal optical properties, Crystals 7, 1 (2017).

[48] C. F. Bohren, D. R. Huffman, and E. E. Clothiaux, Absorption and Scattering of Light by Small Particles (Wiley-VCH, Weinheim, Germany, 2009).

[49] Lumerical Solutions, Inc., http://www.lumerical.com/tcadproducts/mode/. 